\title{
Rare Tumour - Sebaceous Carcinoma of the Scalp
}

\author{
VimalReddy K. ${ }^{1}$, Yohesuwary G. ${ }^{2}$ \\ Department of General Surgery, Hospital TelukIntan, Perak, Malaysia
}

\begin{abstract}
Sebaceous carcinoma (SC) is a rare carcinoma arising from sebaceous gland divided into ocular and extra ocular. Ocular SC is more common than extra ocular. This tumour has a high incidence of recurrence and metastasis. Due to its rarity, it is often difficult to arrive to a diagnosis. Early diagnosis and treatment is crucial. Definitive diagnosis is only by histopathological examination. We are presenting a case of sebaceous carcinoma arising from the scalp.
\end{abstract}

\section{Introduction}

Sebaceous carcinoma (SC) is a rare aggressive malignant tumour derived from the adnexal epithelium of sebaceous glands. It is most commonly seen in the orbital region(2). Extra orbital sebaceous cell carcinoma is extremely rare which commonly occurs on head and scalp due to the abundant sebaceous glands(3). Most sebaceous gland carcinomas have no obvious aetiology and only a few are associated with Muir-Torre syndrome(MTS). This tumour is thought to arise from sebaceous glands in the skin and thus, may crop up anywhere in the body, where these glands exist including the genitalia. SCtraditionally classified into 2 groups, those arising from the ocular adnexa, particularly the Meibomian glands and glands of Zeiss, and those arising in extra ocular sites(4). Ocular SC represents $0.2 \%$ to $0.8 \%$ of all eyelid tumours and $1 \%$ to $5.5 \%$ of all eyelid malignancies(6). It is estimated that approximately $25 \%$ of all reported cases of sebaceous carcinoma occur in extra ocular site. It has been reported that more than $70 \%$ of sebaceous carcinomas are located in the head and neck regions, where sebaceous glands are most commonly located (5).These tumours have a high incidence of local recurrence and regional metastasis. Earlydiagnosis is crucial in reducing the morbidity and mortality associated with the tumour(6). This is a case report of an elderly manwith a sebaceous carcinoma of the scalp.

\section{Case Report}

A 67 years old man, with underlying diabetes, hypertension and chronic kidney disease presented to the surgical outpatient department with a painless swelling over the occiput for 2 years which was gradually increasing in size. There was no discharge, fever, constitutional symptoms or any family history of malignancy. Therewere no other swellings elsewhere. On examination, there was a pedunculated mass over the occiput measuring $3 \times 2 \mathrm{~cm}$ with no signs of inflammation or tenderness. It was mobile and cystic in consistency. No regional lymph nodes were palpable and systemic examination wasunremarkable. His routine blood investigations were within normal limits. Patient underwent an excision biopsy under local anaesthesia whereby the histopathological examination of the swelling came back as sebaceous carcinoma, moderately differentiated grade II, close to excision margin $<1 \mathrm{~mm}$.Macroscopically, there was polypoidal skin covered mass measuring $35 \mathrm{~mm}$. The cut section shows multilobulated solid whitish mass $(35 \times 30 \times 30 \mathrm{~mm})$ with few cystic spaces containing gelatinous material. The umbilicated mass show central yellowish of area of necrosis.Microscopically,the cut section were composed of large polygonal tumourcells, with abundant cytoplasm and oval vesicular nuclei with distinctnucleoli.Sebocyticdifferentiation frequently seen with mitoses. Immunohistochemistry tests showed the tumour cells are focally positive for EMA and negative for S-100, CEA, BCL2.

Due to the close to excision margins, patient was planned for re-excision of tumour margin whereby margins of $5 \mathrm{~mm}$ was excised and sent for histopathological examination which came back as negative for residual tumor. Subsequently, a CT scan of the head, neck, thorax and abdomen was done which showed no evidence of distant metastasis. He was referred to the oncology clinic whereby no further treatment was required at this moment of time. The patient was planned to be seen in the surgical outpatient department in 2 months upon discharge and for a surveillance colonoscope to rule out visceral malignancies.

\section{Discussions}

Sebaceous carcinoma is a rare malignant neoplasm, often occurs in adults with a slight male preponderance. This malignancy can occur as peri-ocular and extra ocular types and the former variant contributes to $75 \%$ of sebaceous neoplasms(7). Extra orbital SC has a different behaviour compared to the orbital SC which is considered less aggressive than orbital $\mathrm{SC}(3)$. This tumour is thought to arise from sebaceous gland in the skin and thus, may arise anywhere in the body where these glands exist. (1) It may 
appear on the top of pre-existing dermatosis, such as nevus sebaceous and actinic keratosis or may follow radiation therapy for other diseases. It may also occur in Muir-Torre syndrome, characterized by occurrence of sebaceous tumours in association with visceral malignancies(1). It is shown to be related to germ line mutations in the MSH2 and MLH1 genes found on chromosomes 3p and 2p, respectively(5). Reported risk factors for sebaceous carcinoma include advanced age,Asian or South Asian race, women, previous irradiation to the head and neck and a genetic predisposition for Muir-Torre syndrome or possibly familial retinoblastoma(8).

Since SC presents most commonly as a gradually enlarging, firm nodule, it can mimic more common ophthalmologic or dermatologic conditions. Definitive diagnosis is only by pathology. Involvement of preauricular or cervical lymph nodes has been associated previously with a $50 \%$ to $67 \%$-year mortality rate.Extra ocular SC and smaller tumor sizes are associated with lower metastatic potential and consequently, lower mortality $(8)$. Due to the variety of clinical presentations and histological growth patterns the diagnosis is often delayed for months to years. The clinical appearance of extra ocular sebaceous carcinoma is not pathognomic, but the lesion may be a pink to red-yellow nodule. The possibility of MTS must be considered in every case of sebaceous tumor.

Criteria for diagnosis of Muir-Torre syndrome include the presence of at least one sebaceous adenoma, sebaceous epitheliomaor sebaceous carcinoma and at least one visceral cancer(1). SC histologically may be classified as well, moderately or poorly differentiated. The morphological hall mark of sebaceous differentiation is the detection of sebaceous cells and demonstration of fat in vacuolated tumorcells(7). Poorly differentiated sebaceous carcinomas lack lipid vesicles and consist of predominantly basaloidtumor cells that imitate carcinomas such as basal cell carcinoma and squamous cell carcinoma(5). The histological criteria for sebaceous carcinoma are high mitotic activity, nuclear pleomorphism, lobular architecture and foamy vacoulization of the cytoplasm [2].Immunohistochemically, the tumor cells of SC show positive reactions for EMA and Ber-EP4, in contrast to squamous cell carcinoma which are negative for Ber-EP4. Human milk fat globules subclass 1 and 2 (HMFG1 and HMFG2) are positive in basal cell carcinoma which helps it to distinguish it from sebaceous cell carcinoma in which it is usually negative (1). Histologically, the poor prognostic indicators are poor differentiation, presence of lymphatic or vascular permeation, presence of pagetoid cells shown in histology and immuno-histochemical staining (2).

In contrast to basal cell carcinoma, SC can be an aggressive form of cancer. Local recurrence rate after surgical excision within 5 years is found to be approximately $9 \%$ to $36 \%$ in sebaceous carcinoma and $10.1 \%$ in basal cell carcinoma. Furthermore, metastasis occurs at relatively higher rate than that observed basal cell carcinoma(5). Distant metastases and recurrence rates are more common in the ocular type of sebaceous carcinoma when compared to extra ocular sebaceous carcinoma. Mortality rates irrespective of ocular or extra ocular type ranges from $9 \%$ to 50\%.[7).The primary treatment of sebaceous carcinoma is a wide excision with a safety margin of $5 \mathrm{~mm}$ to $10 \mathrm{~mm}$. Radiotherapy or chemotherapy has been the treatment of choice with postoperative care particularly when metastasis is confirmed.(5).

\section{Conclusion}

In conclusion, sebaceous carcinoma is a rare, aggressivetumour which provides a diagnostic dilemma for many physicians and surgeons. Diagnosis is only achieved via histopathological examination. Accurate and prompt diagnosis is crucial and a clear margin of at least $1 \mathrm{~cm}$ is needed. Besides that,thistumor should alert the clinician to the possibility of Muir-Torre syndrome. Patients must be followed up for possibility of recurrence.

\section{References}

[1]. Bhatia SK, Atri S, Anjum A, Sardha M, Ali SA, Zaheer S, et al. Postauricular sebaceous cell carcinoma. International Journal of Case Reports and Images 2012;3(9):29-32.

[2]. Mathur S K, Singh Sunita, Yadav Rajni, Duhan Amrita, Sen Rajeev.Extraocular Sebaceous Carcinoma - a Rare Tumour at a Rare Site.Egyptian Dermatology Online Journal 2010; 6(2):14.

[3]. Bhavarajua VMK, Shamim SE, Naik VR, Shaari S. Sebaceous cell carcinoma of scalp - Arare presentation. Malaysian Journal of Medical Sciences 2007; 14(1): 67-70.

[4]. Ghosh SK, Bandyopadhyay D, Gupta S, Chatterjee G, Ghosh A. Rapidly growing extraocular sebaceous carcinoma occurring during pregnancy: A case report. DermatologyOnline Journal 2008; 14(8): 8.

[5]. JoonHo Lee, Hea-Kyeong Shin, and Tae Jung Jang. A Case of Rapidly GrowingExtraocular Sebaceous Carcinoma 2014 Apr 15(1):32-35.

[6]. Tan O, Ergen D, and Arslan R. Sebaceous Carcinoma on the Scalp. Dermatologic Surgery, 2006; 32: 1290-1293.

[7]. Karthika Natarajan, Reena Rai, and Suma B. Pillai.Extra ocular sebaceous carcinoma: A rare case report. Indian Dermatology Online Journal, 2011 Jul-Dec; 2(2): 91-93.

[8]. Dasgupta T, Wilson L.D. and Yu, J.B. A retrospective review of 1349 cases of sebaceous carcinoma. Cancer 2009; 115:158-65. 